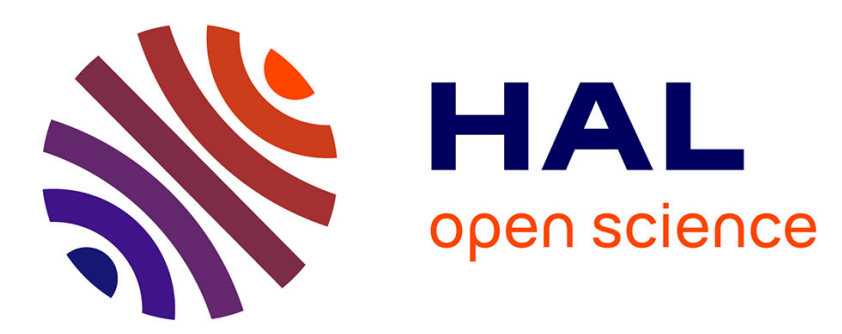

\title{
Influence of Temperature and/or Field Dependences of the E-J Power Law on Trapped Magnetic Field in Bulk $\mathrm{YBaCuO}$
}

Kévin Berger, Jean Lévêque, Denis Netter, Bruno Douine, Abderrezak Rezzoug

\section{To cite this version:}

Kévin Berger, Jean Lévêque, Denis Netter, Bruno Douine, Abderrezak Rezzoug. Influence of Temperature and/or Field Dependences of the E-J Power Law on Trapped Magnetic Field in Bulk YBaCuO. IEEE Transactions on Applied Superconductivity, 2007, 17 (2), pp. 3028-3031. 10.1109/TASC.2007.902095 . hal-00348340

\section{HAL Id: hal-00348340 \\ https://hal.science/hal-00348340}

Submitted on 18 Dec 2008

HAL is a multi-disciplinary open access archive for the deposit and dissemination of scientific research documents, whether they are published or not. The documents may come from teaching and research institutions in France or abroad, or from public or private research centers.
L'archive ouverte pluridisciplinaire HAL, est destinée au dépôt et à la diffusion de documents scientifiques de niveau recherche, publiés ou non, émanant des établissements d'enseignement et de recherche français ou étrangers, des laboratoires publics ou privés. 


\title{
Influence of temperature and/or field dependences of the $E-J$ power law on trapped magnetic field in bulk $\mathrm{YBaCuO}$
}

\author{
K. Berger, J. Lévêque, D. Netter, B. Douine, and A. Rezzoug
}

\begin{abstract}
Temperature, current density and magnetic field distributions in YBCO bulk superconductor during a pulsed-field magnetization (PFM) process are calculated using the finite difference method. Simulations are based on the heat diffusion equation with account of the heat produced by flux motion, and Maxwell's equations. A power law with temperature and magnetic field dependent parameters is used to characterize the electromagnetic behavior of the superconducting material. We analyze how the stored magnetic energy $W_{m}$ depends on the temperature and field dependences of the power law.
\end{abstract}

Index Terms - bulk YBaCuO, stored magnetic energy, thermal coupling, magnetization, modelling.

\section{INTRODUCTION}

$\mathrm{T}_{\mathrm{h}}$ HE recent developments in preparing melt-textured high-temperature superconductor (HTS) bulk parts with high critical current density, close to $10^{9} \mathrm{~A} \cdot \mathrm{m}^{2}$ at $77 \mathrm{~K}$, make this material particularly promising for use as cryo-permanent magnets [1], [2].

To magnetize the HTS, pulsed field magnetization (PFM) process is often used because it is considered to be the most practical method. However, this process leads to a strong temperature rise in the superconductor produced by flux motion and it is found experimentally that trapped flux reaches a maximum at some optimal value $B_{\text {opt }}$ of the maximal applied field $B_{m}$, and decreases for larger $B_{m}$ [3].

In this paper, numerical simulations are performed to obtain and understand electromagnetic and thermal phenomena in an YBCO bulk part during PFM. The coupled magneto-thermal problem to be solved is 2D-axisymmetrical and strongly nonlinear. Simulations are based on the Finite Difference Method and allow for the calculation of the magnetic field, current density and temperature distributions in the superconductor during all the stages of the PFM process.

In order to have a more global idea of the trapped field inside the HTS part, we chose to study the stored magnetic

Manuscript submitted August 24, 2006.

The authors are with the Research Group in Electrical engineering and Electronics of Nancy, University of Nancy, BP 239, F-54506 Vandoeuvre-lèsNancy, France (corresponding author to provide phone: +33 (0)3 836841 27; fax: +33 (0)3 836841 33; e-mail: Kevin.Berger@green.uhp-nancy.fr; Jean.Leveque@green.uhp-nancy.fr; Denis.Netter@green.uhp-nancy.fr; Bruno .Douine@green.uhp-nancy.fr; Abderrezak.Rezzoug@green.uhp-nancy.fr). energy in the superconductor. We will show that only the taking into account of the heat generation can reproduce experimental results showing a maximum in the stored magnetic energy.

\section{NUMERICAL ANALYSIS METHOD}

\section{A. Magnetization process}

In the PFM experiment, a cylindrical bulk YBCO (Fig. 1) is magnetized by using a pulsed $\mathrm{Cu}$ coil which, together with a capacitor, form a resonant circuit. The resulting applied field $B_{a}(t)$ in the $z$ direction can be expressed as:

$B_{a}(t)=B_{m} \frac{t}{\tau} \exp \left(1-\frac{t}{\tau}\right)$,

with $B_{m}$ the maximal applied field at $t=\tau$, and $\tau$ the time constant of the system. In order to simulate a PFM process, we chose a time constant $\tau$ of 1 ms. Fig. 2 shows the temporal evolution of the applied magnetic field $B_{a}(t)$.

\section{B. Model description}

The electromagnetic behavior of a superconductor is governed by the Maxwell's equations. When quasi-static approximation is taken (no displacement currents are considered and $\nabla \cdot \mathbf{J}=0$ ), the supplement of the constitutive

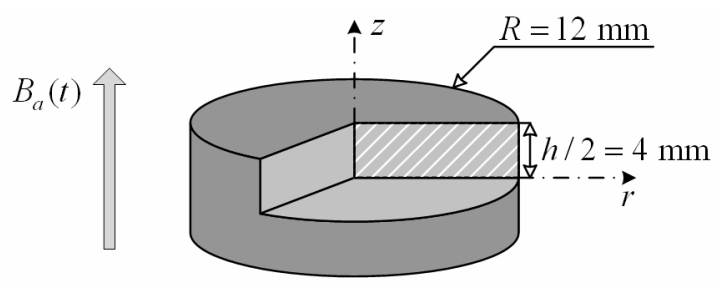

Fig. 1. Sample geometry: compact cylinder with $12 \mathrm{~mm}$ of outer radius and $8 \mathrm{~mm}$ of height. Due to symmetry only one half of the sample is modeled (hatched part).

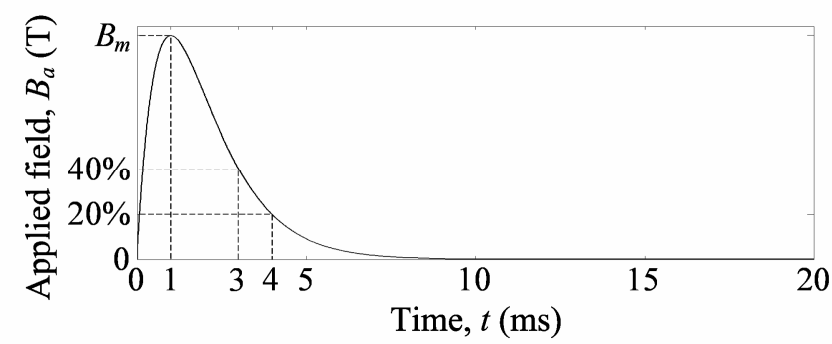

Fig. 2. Temporal evolution of the applied magnetic field $B_{a}(t)$. 
law $\mathbf{B}=\mu_{0} \mathbf{H}$ gives:

$\nabla \times \nabla \times \mathbf{E}=-\mu_{0} \partial \mathbf{J} / \partial t$.

Because the superconductor is subjected to a non-stationary external magnetic field $B_{a}(t)$, a heat per unit volume is produced:

$Q=\mathbf{E} \cdot \mathbf{J}$.

The temperature change due to the heat generation is described by the heat diffusion equation:

$\gamma C \frac{\partial T}{\partial t}-\nabla \cdot(\kappa \nabla T)=Q$,

where $T$ is the temperature, $\gamma$ the density, $C$ the specific heat capacity, and $\kappa$ the thermal conductivity.

The heat transfer is considered essentially by convection between the superconductor and the liquid nitrogen bath. In this liquid, the convective heat transfer coefficient $h^{*}$ is approximately $400 \mathrm{~W} /\left(\mathrm{m}^{2} \cdot \mathrm{K}\right)$ according to [4].

In axially symmetric problems the electric field $\mathbf{E}$ and the current density $\mathbf{J}$ have only a non-zero component in the $\theta$ direction, $E$ and $J$. Then the fundamental equations on the axisymmetric coordinate $(r, z)$ are as follows:

$\frac{1}{r} \frac{\partial E}{\partial r}+\frac{\partial^{2} E}{\partial r^{2}}-\frac{E}{r^{2}}+\frac{\partial^{2} E}{\partial z^{2}}=\mu_{0} \frac{\partial J}{\partial t}$,

$\gamma C \frac{\partial T}{\partial t}=\frac{\kappa}{r} \frac{\partial T}{\partial r}+\kappa \frac{\partial^{2} T}{\partial r^{2}}+\kappa \frac{\partial^{2} T}{\partial z^{2}}+Q$.

The power law model is used to describe the nonlinear $E-J$ characteristic of the superconductor,

$E=E_{c}\left(J / J_{c}\right)^{n}$,

where $J_{c}$ is the critical current density, $E_{c}$ the critical electrical field, and $n$ the index of the power law. The parameters $J_{c}$ and $n$ can also depend on the temperature $T$ and the magnetic flux density B. In Kim's model [5] the flux density dependence of $J_{c}$ is considered as:

$J_{c}(\mathbf{B})=J_{c 0} \frac{1}{1+\|\mathbf{B}\| / B_{0}}$,

where $J_{c 0}$ is $J_{c}$ for $\|\mathbf{B}\|=0$ and $B_{0}$ a constant. The field dependence model of the $n$-value is taken as:

$n(\mathbf{B})=n_{1}+\frac{n_{0}-n_{1}}{1+\|\mathbf{B}\| / B_{0}}$,

where $n_{0}$ and $n_{1}$ are respectively $n$-value for $\|\mathbf{B}\|=0$ and $\|\mathbf{B}\|>>B_{0}$.

On the basis of Anderson's excitation creep model, the index $n$ can be identified with $U_{0 a} / k_{\mathrm{B}} T$, where $U_{0 a}$ is the apparent pinning potential [7], and $k_{\mathrm{B}}$ the Boltzmann's constant. Measurements of $J_{c}$ as a function of $T$ have successfully been fitted to linear model in [7]. The following expressions are used:

$J_{c}(T)=J_{c 0} \frac{1-T / T_{c}}{1-T_{0} / T_{c}}$,

$n(T)=n_{0} T_{0} / T$ with $n_{0}=U_{0 a} / k_{\mathrm{B}} T_{0}$.

Here $T_{0}$ is the liquid nitrogen bath temperature, $J_{c 0}$ and $n_{0}$ are respectively the critical current density and the $n$-value for the case $T=T_{0}$.

Four $E-J$ laws with or without thermal and magnetic field
TABLE I

RELATIONSHIPS BETWEEN $E, J, \mathbf{B}$ AND $T$

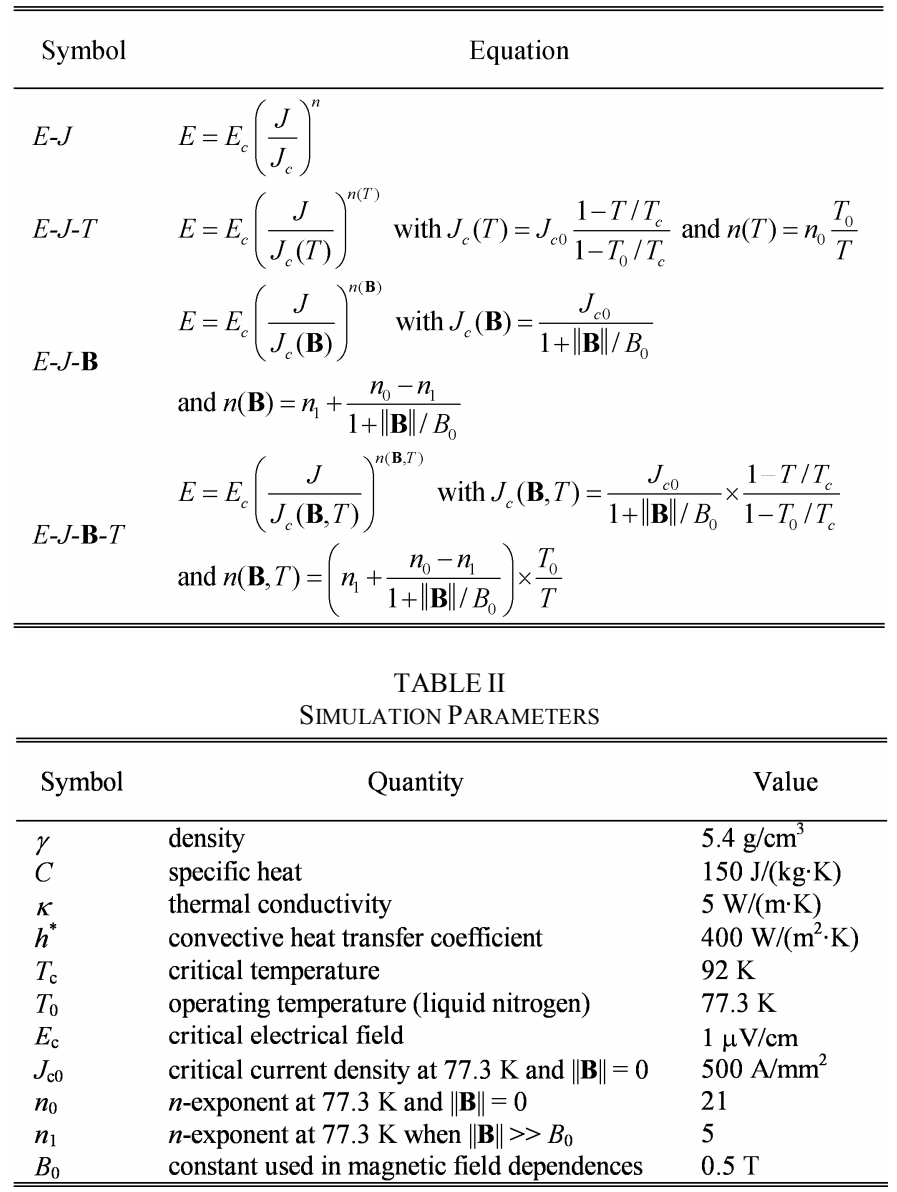

dependences are chosen for studying the electromagnetic and thermal phenomena in the bulk YBCO during PFM (Table I).

\section{RESULTS}

We present the results of the calculations assuming a sample (Fig. 1) characterized by parameters resumed in Table II, which are typical values for $\mathrm{YBa}_{2} \mathrm{Cu}_{3} \mathrm{O}_{7-\delta}$ crystal. Due to symmetry, results are only shown in the hatched part of the sample.

Magnetic field and current density distributions obtained using the different $E-J$ laws are compared at different times in Fig. 3 and Fig. 4 respectively. From $t=0$ to $1 \mathrm{~ms}$, the applied field $B_{a}$ increases up to $B_{m}=5 \mathrm{~T}$ and screening currents are induced from the edge of the superconductor (Fig. 4). Because $B_{m}$ is lower than the full penetration field $B_{p}=\mu_{0} J_{c} R \approx 7.5 \mathrm{~T}$, the magnetic field doesn't penetrate to the center of the YBCO cylinder in the case $E-J$. On the contrary, the other laws with thermal or magnetic field dependence lead to a faster penetration of the field and, consequently, smaller current-free region in the center.

From 1 to $20 \mathrm{~ms}, B_{a}$ decreases down to zero and areas with opposite current appear. At $t=3 \mathrm{~ms}$, the applied field is about $40 \%$ of $B_{m}$, i.e. 2 T.

From $20 \mathrm{~ms}$ to $200 \mathrm{~s}$, the applied magnetic field does not change anymore. However, we can see that trapped magnetic 
field and current density distributions change. The first reason for $E-J-T$ and $E-J-\mathbf{B}-T$ laws is that thermal effects are considered and the displacement of the hot spot creates region with lower current density. The second reason is not thermal, the change of the electromagnetic quantities are due to relaxation phenomena generated by the power law $E(J)$.
The main difference between $E-J$ and $E-J$-B laws is that the saturating current density in the latter is varying with the local field amplitude - the higher the local field, the lower the current density. This is also true for $E-J-\mathbf{B}-T$ law but thermal effects have to be considered too.

Heat produced by flux motion has been calculated for each

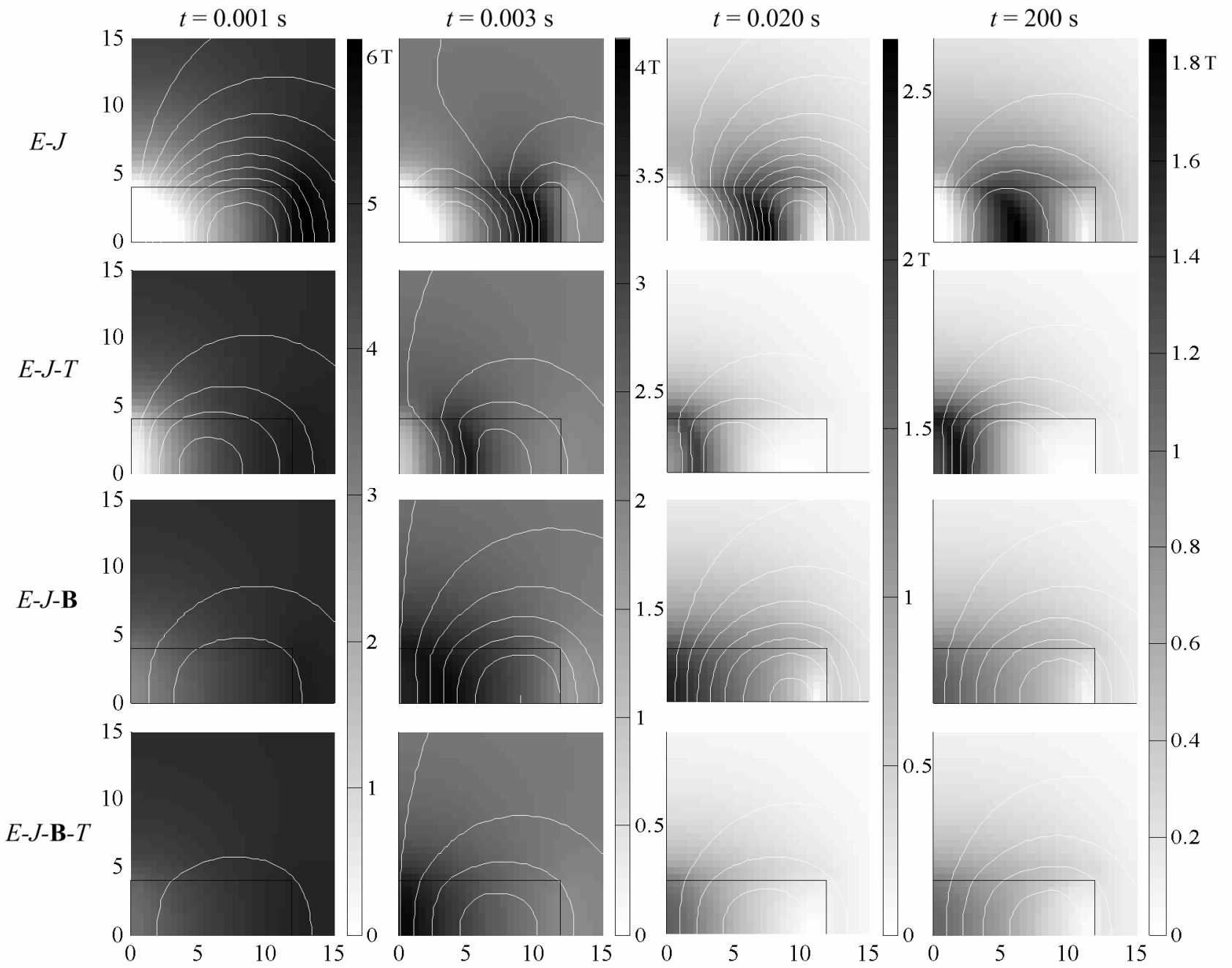

Fig. 3. Comparison of the different $E-J$ laws. Magnetic field distributions $\mathbf{B}(r, z)$ during PFM with $B_{m}=5 \mathrm{~T}$ and $\tau=1 \mathrm{~ms}$. Due to symmetry, results are shown in the top right half of the sample (Fig. 1). Sample boundaries are situated at $r=12 \mathrm{~mm}$ and $z=4 \mathrm{~mm}$.

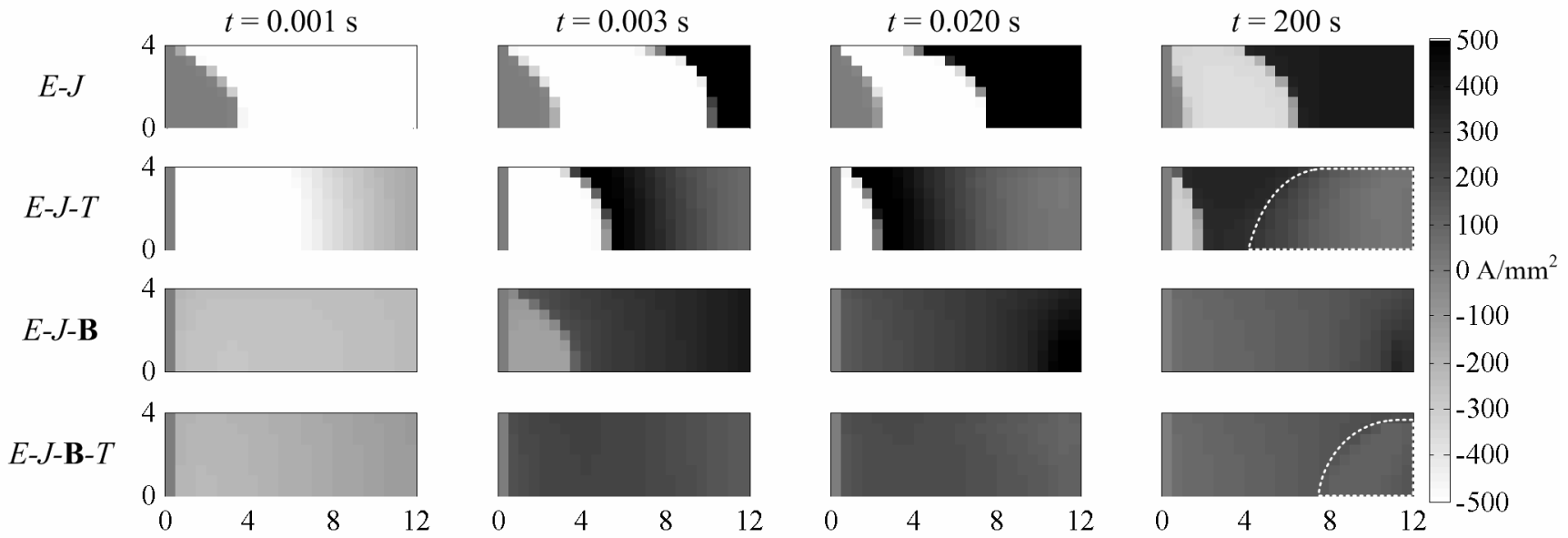

Fig. 4. Comparison of the different $E-J$ laws. Current density distributions $J(r, z)$ during PFM with $B_{m}=5 \mathrm{~T}$ and $\tau=1$ ms. Due to symmetry, results are shown in the top right half of the sample (Fig. 1). 

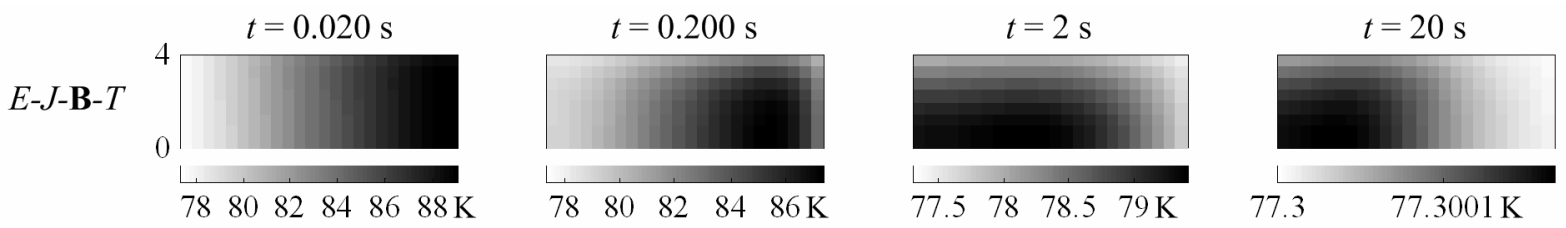

Fig. 5. Displacement of the hot spot. Temperature distributions $T(r, z)$ during PFM with $B_{m}=5 \mathrm{~T}$ and $\tau=1 \mathrm{~ms}$ in the case $E-J-\mathbf{B}-T$. Due to symmetry, results are shown in the top right half of the sample (Fig. 1).

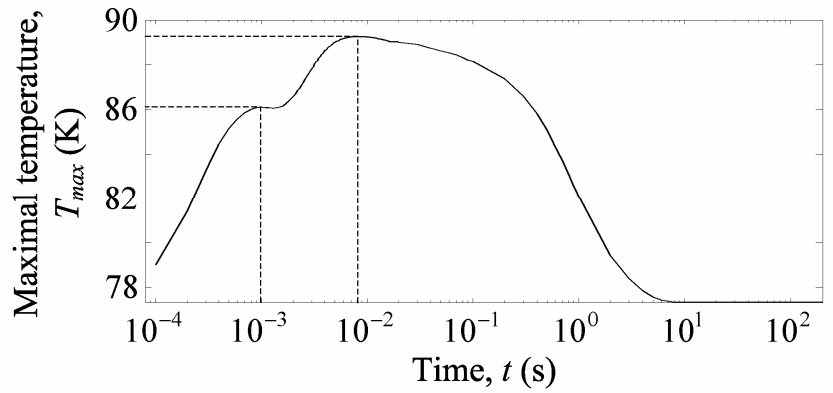

Fig. 6. Maximal temperature in the bulk YBCO as a function of time in the case $E-J-\mathbf{B}-T$.

case but only $E-J-T$ and $E-J-\mathbf{B}-T$ laws depend on temperature. Temperature distributions with $E-J$-B- $T$ law are shown in Fig. 5. Approximately the same results have been obtained with $E$ $J-T$.

Fig. 6 shows the maximal temperature in the bulk YBCO as a function of time. Because of the thermal time constant, we have to wait several seconds in order to obtain the magnetothermal steady state. We assume that the superconductor is in steady state at $t=200 \mathrm{~s}$.

From 0 to $20 \mathrm{~ms}$, the applied magnetic field varies and temperature distributions present very clear maxima near the sample surface. The reason is obvious as near the surface the flux motion is most intense and the heat release is maximal. In fact, the maximal temperature in the superconductor is observed at the edge of the superconductor at $t=8 \mathrm{~ms}$ (Fig. 6). After this time, the heat is diffusing from the edge to the center of the bulk YBCO (Fig. 5). Therefore, with $E-J-T$ and $E-J-\mathbf{B}-T$ laws at $t=200 \mathrm{~s}$, we can see an area (inside the dotted lines in Fig. 4) where the current density is lower.

When $\tau$ is given, the key point to magnetize the superconductor is to choose the optimum applied field $B_{m}$ so that in the remanent state, the trapped magnetic field becomes maximum. The stored magnetic energy defined as,

$W_{m}=\frac{1}{2} \iiint_{\mathbb{R}^{3}} \mathbf{H} \cdot \mathbf{B} \cdot d v=\frac{1}{2} \iiint_{\Omega_{s}} \mathbf{A} \cdot \mathbf{J} \cdot d v$

is plotted in Fig. 7 as a function of $B_{m}$. Here, $\Omega_{s}$ is the volume of superconductor, and $\mathbf{A}$ the magnetic vector potential.

With the $E-J$ law, the saturating current density is neither affected by magnetic field nor temperature. Therefore, the predicted stored magnetic energy is the highest with this model. The maximal value (not represented) of $4.7 \mathrm{~J}$ is reached for $B_{m}=11 \mathrm{~T}$. When taking into account the magnetic field dependence, the penetration field as well as the current density are lower, which results in a lower maximum of the stored energy. For $E-J-T$ and $E-J-\mathbf{B}-T$ laws, the presence of heating leads to reduction of the stored magnetic energy, and a

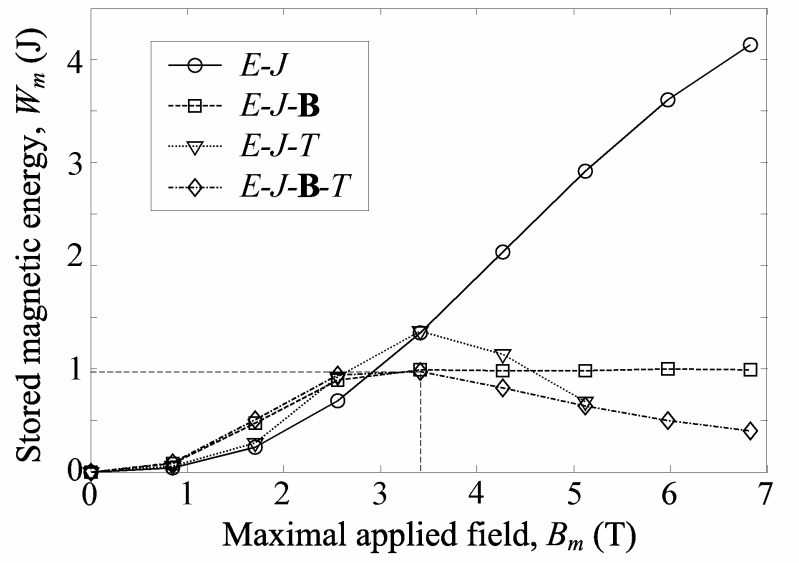

Fig. 7. Comparison of the different $E-J$ laws. Stored magnetic energy $W_{m}$ as a function of the maximal applied field $B_{m}$.

peak in the $W_{m}\left(B_{m}\right)$ curve appears at $B_{m}=3.5 \mathrm{~T}$.

\section{CONCLUSION}

Through this article, the importance of temperature and field dependences of the $E-J$ power law on the superconductor's behavior during a PFM process was shown. The stored magnetic energy $W_{m}$ with the $E-J-\mathbf{B}-T$ law, which reproduces the most realistic behavior, reaches maximum for some optimal maximum field $B_{\text {opt }}$. The value of $B_{\text {opt }}$ can be found theoretically, which enables the design of more efficient pulsed system to magnetize cryo-magnets.

\section{REFERENCES}

[1] M. Muralidhar, M. R. Koblischka and M. Murakami, "Effect of platinum addition on the microstructure and critical current density in (Nd, Eu, Gd)-Ba-Cu-O," Superconductor Science and Technology 12, pp. 105-109, 1999.

[2] S. Nariki, N. Sakai, M. Murakami and I. Hirabayashi, "High critical current density in $\mathrm{Y}-\mathrm{Ba}-\mathrm{Cu}-\mathrm{O}$ bulk superconductors with very fine Y211 particles," Supercond. Sci. Technol. 17, pp. S30-S35, 2004.

[3] U. Mizutani, H. Ikuta, T. Hosokawa, H. Ishihara, K. Tazoe, T. Oka, Y. Itoh, Y. Yanagi and M. Yoshikawa, "Applications of superconducting permanent magnets driven by static and pulsed fields," Supercond. Sci. Technol. 13, pp. 836-840, 2000.

[4] M. Polák, J. Kvitkovič, E. Demenčík, L. Janšák, P. Mozola, "Temperature of Bi-2223/Ag samples in the resistive section of I-V curves," Physica C, vol. 401, pp. 160-164, 2004.

[5] Y. B. Kim, C. F. Hempstead, and A. R. Strnad, "Critical Persistent Currents in Hard Superconductors," Physical Review Letter 9, pp. 306309, 1962.

[6] L. Cesnak, F. Gömöry, P. Kováč, "Pinning potential, its relativity and dependence on temperature and magnetic field studied on the basis of the I-V characteristics of multifilamentary Nb3Sn superconductors," Supercond. Sci. Technol. 9, pp. 184-192, 1996.

[7] N. Schönborg, S. Hörnfeldt, "Model of the temperature dependence of the hysteresis losses in a high-temperature superconductor," Physica C, vol. 372-376, pp. 1734-1738, 2002. 